\title{
$\beta$-Mangostin induces p53-dependent G2/M cell cycle arrest and apoptosis through ROS mediated mitochondrial pathway and NfkB suppression in MCF-7 cells
}

\begin{abstract}
$\beta$-Mangostin $(\beta M)$ was isolated from Cratoxylum arborescens to investigate its anti-cancer effect in MCF-7 cells. $\beta \mathrm{M}$ induced apoptosis by down-regulation of $\mathrm{Bcl} 2$ and up-regulation of Bax, triggering the cytochrome c release from mitochondria to cytosol. The release of caspase-9 and -7 and consequently cleaved PARP leading to apoptotic was observed upon treatment. Reduction of both bid and caspase 8 and the up regulation of Fas showed the involvement of the extrinsic pathway. Significantly up regulated GADD45A and HRK genes were observed upon treatment, with concomitant inhibition of NF-kB to nucleus. The protein array had demonstrated the expression of HSP 70, HSP 60, XIAP, Survivin, p53 and Bax. Moreover, $\beta \mathrm{M}$ had showed p53-dependent G2/M cell cycle arrest by down regulation of cdc2 and PCNA. Together, the results demonstrated that the $\beta \mathrm{M}$ induced anti-proliferative effect, leading to G2/M phase cell cycle arrest and apoptosis through both the extrinsic and mitochondrial pathways with the involvement of the multiple pro and anti-apoptosis and NF$\mathrm{kB}$ signalling pathways.
\end{abstract}

Keyword: Cratoxylum arborescens; $\beta$-Mangostin; Apoptosis; p53; NF-kB; Bax/Bcl-2 